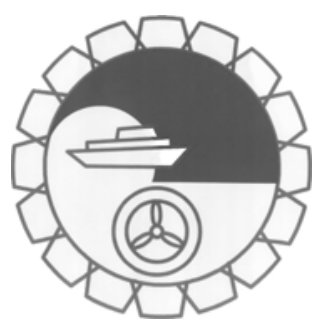

ournal of Naval Architecture and Marine Engineering

\title{
DUFOUR AND SORET EFFECTS ON STEADY MHD FREE CONVECTION AND MASS TRANSFER FLUID FLOW THROUGH A POROUS MEDIUM IN A ROTATING SYSTEM
}

Nazmul Islam and Md. Mahmud Alam

Mathematics Discipline, Khulna University, Khulna-9208, Bangladesh, E-mail: alam_mahmud2000@yahoo.com

\begin{abstract}
The numerical studies are performed to examine the steady MHD free convection and mass transfer fluid flow through a continuously moving porous medium with thermal diffusion and diffusion thermo past a semi-infinite vertical porous plate in a rotating system. Impulsively started plate moving in its own plane is considered. With appropriate transformations the boundary layer equations are transformed into nonlinear ordinary differential equations. The local similarity solutions of the transformed dimensionless equations for the flow, heat and mass transfer characteristics are evaluated using shooting iteration technique. Numerical results are presented in the form of velocity, temperature and concentration within the boundary layer for different parameters entering into the analysis. Also the effects of the pertinent parameters on the local skin-friction coefficients and rate of heat transfer as well as rate of mass transfer in terms of the local Nusselt number and Sherwood number respectively are also discussed.
\end{abstract}

Keywords: MHD free convection, porous medium, rotation effect, Dufour effect, Soret effect.

NOMENCLATURE

\begin{tabular}{|c|c|c|c|}
\hline$x, y, z$ & Cartesian coordinates & $G_{r}$ & Grashof number \\
\hline$f_{w}$ & Transpiration parameter & $G_{m}$ & Modified Grashof number \\
\hline$u, v, w$ & Velocity components & $K$ & $\begin{array}{l}\text { Nondimensional } \\
\text { permeability parameter }\end{array}$ \\
\hline$v_{0}(x)$ & Suction velocity & $P_{r}$ & Prandtl number \\
\hline B & Magnetic field intensity & $S_{c}$ & Schimidt number \\
\hline $\mathbf{B}_{0}(x)$ & $\begin{array}{l}\text { Constant magnetic field } \\
\text { intensity }\end{array}$ & $S_{r}$ & Soret number \\
\hline $\mathbf{J}=\left(J_{x}, J_{y}, J_{z}\right)$ & Current density & $D_{f}$ & Dufour number \\
\hline$\Omega$ & Angular velocity & $M$ & Magnetic parameter \\
\hline $\mathrm{k}$ & $\begin{array}{l}\text { Thermal conductivity of the } \\
\text { medium }\end{array}$ & $R$ & Rotation parameter \\
\hline$g_{0}$ & Gravitational acceleration & $K^{\prime}$ & $\begin{array}{l}\text { Permeability of the porous } \\
\text { medium }\end{array}$ \\
\hline $\mathrm{f}^{\prime}$ & $\begin{array}{l}\text { Non-dimensional primary } \\
\text { velocity }\end{array}$ & $D_{m}$ & $\begin{array}{l}\text { Coefficient of mass } \\
\text { diffusivity }\end{array}$ \\
\hline$g$ & $\begin{array}{l}\text { Non-dimensional secondary } \\
\text { velocity }\end{array}$ & $c_{p}$ & $\begin{array}{l}\text { Specific heat at constant } \\
\text { pressure }\end{array}$ \\
\hline$U_{0}$ & Uniform velocity & $T_{m}$ & Mean fluid temperature \\
\hline
\end{tabular}




\begin{tabular}{|c|c|}
\hline$T$ & Temperature of the flow field \\
\hline$T_{w}$ & Temperature at the plate \\
\hline$T_{\infty}$ & $\begin{array}{l}\text { Temperature of the fluid } \\
\text { outside the boundary layer }\end{array}$ \\
\hline C & Species concentration \\
\hline$C_{w}$ & Concentration at the plate \\
\hline$C_{\infty}$ & $\begin{array}{l}\text { Species concentration outside } \\
\text { the boundary layer }\end{array}$ \\
\hline
\end{tabular}

Greek
$\mu$
$\eta$
$v$
$\theta$
$\varphi$

$\begin{array}{cl}\mathrm{C}_{0} & \text { Mean concentration } \\ k_{T} & \text { Thermal diffusion ratio } \\ c_{s} & \text { Concentration susceptibility } \\ N_{u} & \text { Nusselt number } \\ S_{h} & \text { Sherwood number } \\ \left(\tau_{\mathrm{x}}, \tau_{\mathrm{z}}\right) & \text { Skin-friction coefficients }\end{array}$

$\rho \quad$ Fluid density

$\sigma^{\prime} \quad$ Electrical conductivity

$\beta \quad$ Coefficient of thermal

expansion

$\beta^{*} \quad$ Coefficient of concentration

expansion

\section{Introduction}

The science of magnetohydrodynamics (MHD) was concerned with geophysical and astrophysical problems for a number of years. In recent years the possible use of MHD is to affect a flow stream of an electrically conducting fluid for the purpose of thermal protection, braking, propulsion and control. From the point of applications, model studies on the effect of magnetic field on free convection flows have been made by several investigators. Some of them are Georgantopoulos (1979), Nanousis et al. (1980) and Raptis and Singh (1983). Along with the effects of magnetic field, the effect of transpiration parameter, being an effective method of controlling the boundary layer has been considered by Kafousias (1979) and Singh (1982). On the other hand, along with the free convection currents, caused by the temperature difference, the flow is also effected by the difference in concentrations on material constitution. Gebhart and Pera (1971) made extensive studies of such a combined heat and mass transfer flow to highlight the insight of the flow.

In the above mentioned works, the level of concentration of foreign mass is assumed very low so that the Soret and Dufour effects can be neglected. However, exceptions are observed therein. The Soret effect, for instance, has been utilized for isotope separation, and in mixture between gases with very light molecular weight $\left(H_{2}, H_{e}\right)$ and of medium molecular weight $\left(N_{2}\right.$, air $)$. The Dufour effect was found to be of order of considerable magnitude such that it cannot be ignored (Eckert and Drake, 1972). In view of the importance of above mentioned effects, Kafoussias and Williams (1995) studied the Soret and Dufour effects on mixed free-forced convective and mass transfer boundary layer flow with temperature dependent viscosity. Anghel et al. (2000) vestigated the Dufour and Soret effects on free convection boundary layer flow over a vertical surface embedded in a porous medium. Quite recently, Alam and Rahman (2006) investigated the Dufour and Soret effects on mixed convection flow past a vertical porous flat plate with variable suction.

In consequence of the above studies, several investigators disclosed that the Coriolis force is very significant as compared to viscous and inertia forces occurring in the basic fluid equations. It is generally admitted that the Coriolis force due to Earth's rotation has a strong effect on the hydromagnetic flow in the Earth's liquid core. The study of such fluid flow problem is important due to its applications in various branches of geophysics astrophysics and fluid engineering. In light of, Singh and Singh (1989), Singh (1983,1984) and Raptis and Singh (1985) initiated a few studies by taking various aspects of the flow phenomena. From the point of application in Solar Physics and Cosmic fluid dynamics, it is important to consider the effects of the electromagnetic 
N. Islam and M. M. Alam / Journal of Naval Architecture and Marine Engineering 4(2007) 43-55 and rotation forces on the flow. But no works of the simultaneous effects of the electromagnetic and rotation forces on the hydromagnetic free convection without Dufour and Soret effects have been reported in the literature.

Hence, our objective is to investigate the Dufour and Soret effects on steady MHD free convection and mass transfer flow through a porous medium past a semi-infinite vertical porous plate in a rotating system.

\section{Formulation of the problem and similarity analysis}

Consider a steady MHD free convection and mass transfer flow of an electrically conducting viscous fluid flow through a porous medium past a continuously moving semi-infinite vertical porous plate $y=0$ in a rotating system. The flow is also assumed to be in the $x$-direction which is taken along the plate in the upward direction and $y$-axis is normal to it. Initially the fluid as well as the plate is at rest, after that the whole system is allowed to rotate with a constant angular velocity $\Omega$ about the $y$-axis. The temperature and the species concentration at the plate are constantly raised from $T_{w}$ and $C_{w}$ to $T_{\infty}$ and $C_{\infty}$ respectively, which are thereafter maintained constant. $T_{\infty}$ and $C_{\infty}$ are the temperature and species concentration of the uniform flow respectively. A uniform magnetic field $\mathbf{B}$ is taken to be acting along the $y$-axis which is assumed to be electrically non-conducting. We assumed that the magnetic Reynolds number of the flow is taken to be small enough so that the induced magnetic field is negligible in comparison with applied one (Pai, 1962), so that $\mathbf{B}=\left(0, B_{0}, 0\right)$ and the magnetic lines of force are fixed relative to the fluid. The equation of conservation of charge $\nabla . \mathbf{J}=0$ gives $J_{y}=$ constant, where the current density $\mathbf{J}=\left(J_{x}, J_{y}, J_{z}\right)$. Since the plate is electrically non-conducting, this constant is zero and hence $J_{y}=0$ at the plate and hence zero everywhere. The physical configuration considered here is shown in the following Fig. 1.

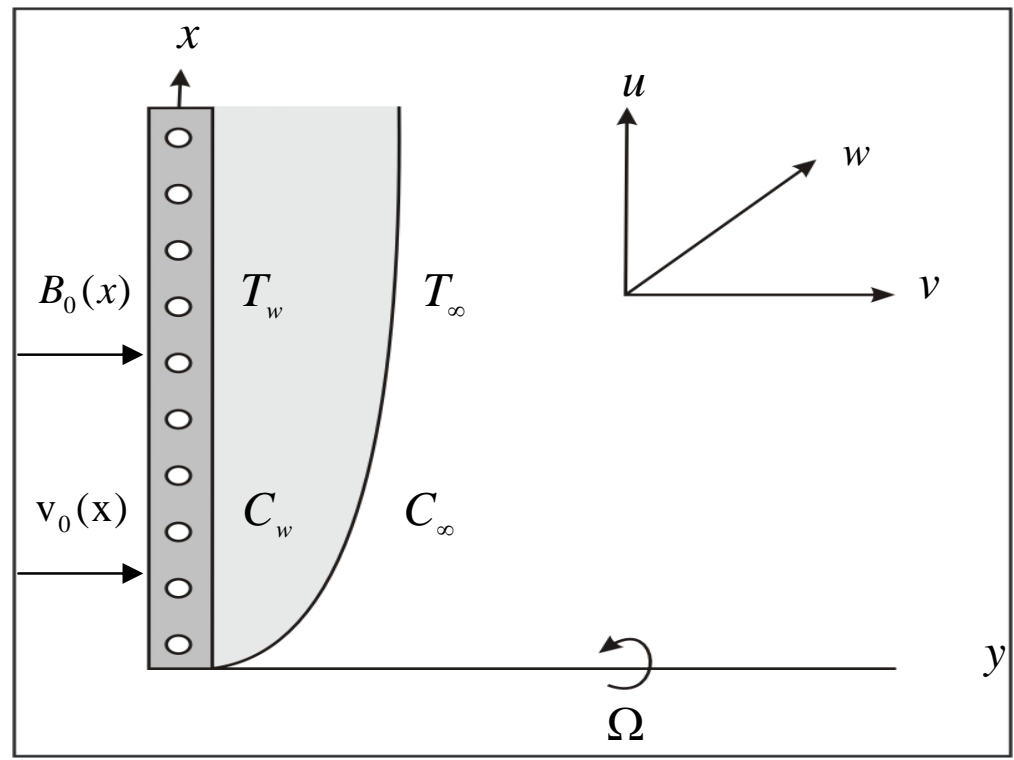

Fig. 1. Physical configuration and coordinate system.

Thus accordance with the above assumptions relevant to the problem and Boussinesq's approximation, the basic boundary layer equations are given by

The continuity equation: $\frac{\partial u}{\partial x}+\frac{\partial v}{\partial y}=0$ 
The momentum equations:

$u \frac{\partial u}{\partial x}+v \frac{\partial u}{\partial y}=v \frac{\partial^{2} u}{\partial y^{2}}+g_{0} \beta\left(T-T_{\infty}\right)+g_{0} \beta^{*}\left(C-C_{\infty}\right)+2 \Omega w-\frac{v}{K^{\prime}} u-\frac{\sigma^{\prime} B_{0}{ }^{2} u}{\rho}$
$u \frac{\partial w}{\partial x}+v \frac{\partial w}{\partial y}=v \frac{\partial^{2} w}{\partial y^{2}}-2 \Omega u-\frac{v}{K^{\prime}} w-\frac{\sigma^{\prime} B_{0}{ }^{2} w}{\rho}$

The energy equation:

$u \frac{\partial T}{\partial x}+v \frac{\partial T}{\partial y}=\frac{k}{\rho c_{p}} \frac{\partial^{2} T}{\partial y^{2}}+\frac{D_{m} k_{T}}{c_{s} c_{p}} \frac{\partial^{2} C}{\partial y^{2}}$

The concentration equation:

$u \frac{\partial C}{\partial x}+v \frac{\partial C}{\partial y}=D_{m} \frac{\partial^{2} C}{\partial y^{2}}+\frac{D_{m} k_{T}}{T_{m}} \frac{\partial^{2} T}{\partial y^{2}}$

The boundary conditions for the present problem are given by:

$\left.\begin{array}{l}u=U_{0}, v=v_{0}(x), w=0, T=T_{w}, C=C_{w} \text { at } y=0 \\ u=0, v=0, w=0, T \rightarrow T_{\infty}, C \rightarrow C_{\infty} \text { at } y \rightarrow \infty\end{array}\right\}$

where all physical quantities are defined in the nomenclature.

We now introduce the following dimensionless variables

$$
\left\{\begin{array}{l}
\eta=y \sqrt{\frac{U_{0}}{2 v x}} \\
f^{\prime}(\eta)=\frac{u}{U_{0}} \\
g(\eta)=\frac{w}{U_{0}} \\
\theta(\eta)=\frac{T-T_{\infty}}{T_{w}-T_{\infty}} \\
\phi(\eta)=\frac{C-C_{\infty}}{\bar{x}\left(C_{0}-C_{\infty}\right)}
\end{array}\right.
$$

Now for reasons of similarity, the plate concentration is assumed to be

$C_{w}(x)=C_{\infty}+\bar{x}\left(C_{0}-C_{\infty}\right)$,

where $C_{0}$ is considered to be mean concentration and $\bar{x}=\frac{x U_{0}}{v}$.

Introducing the relations (7)-(8) into the equations (2)-(5), we obtain the following local similarity equations

$$
\begin{aligned}
& f^{\prime \prime \prime}+f f^{\prime \prime}+G_{r} \theta+G_{m} \phi-K f^{\prime}-M f^{\prime}-2 R g=0 \\
& g^{\prime \prime}+f g^{\prime}-K g-M g+2 R f^{\prime}=0 \\
& \theta^{\prime \prime}+P_{r} f \theta^{\prime}+P_{r} D_{f} \phi^{\prime \prime}=0 \\
& \phi^{\prime \prime}-2 S_{c} f^{\prime} \phi+S_{c} f \phi^{\prime}+S_{c} S_{r} \theta^{\prime \prime}=0
\end{aligned}
$$


where $G_{r}=\frac{g_{0} \beta\left(T_{w}-T_{\infty}\right)}{U_{0}{ }^{2}} 2 x, G_{m}=\frac{g_{0} \beta^{*}\left(C_{0}-C_{\infty}\right)}{v U_{0}} 2 x^{2}$,

$K=\frac{2 v x}{K^{\prime} U_{0}}, M=\frac{\sigma^{\prime} B_{0}^{2} 2 x}{\rho U_{0}}, R=\frac{2 \Omega x}{U_{0}}, P_{r}=\frac{\rho v c_{p}}{k}, D_{f}=\frac{D_{m} k_{T}}{C_{s} c_{p} v} \frac{x U_{0}}{v} \frac{\left(C_{0}-C_{\infty}\right)}{\left(T_{w}-T_{\infty}\right)}$,

$S_{c}=\frac{v}{D_{m}}$ and $S_{r}=\frac{D_{m} k_{T}}{v T_{m}} \frac{v}{x U_{0}} \frac{\left(T_{w}-T_{\infty}\right)}{\left(C_{0}-C_{\infty}\right)}$.

The corresponding boundary conditions are

$$
\left.\begin{array}{l}
f=f_{w}, f^{\prime}=1, g=0, \theta=1, \phi=1 \text { at } \eta=0 \\
f^{\prime}=0, g=0, \theta=0, \phi=0 \text { as } \eta \rightarrow \infty
\end{array}\right\}
$$

where $f_{w}=-v_{0}(x) \sqrt{\frac{2 x}{v U_{0}}}$ is taken to be transpiration parameter.

\section{Skin-friction coefficients, Nusselt number and Sherwood number}

The quantities of physical interest are the skin friction coefficients, the Nusselt number and the Sherwood number. The equations defining the wall skin frictions are

$\tau_{x}=\mu\left(\frac{\partial u}{\partial y}\right)_{y=0}, \tau_{z}=\mu\left(\frac{\partial w}{\partial y}\right)_{y=0}$ which are proportional to $\left(\frac{\partial^{2} f}{\partial \eta^{2}}\right)_{\eta=0}$ and $\left(\frac{\partial \mathrm{g}}{\partial \eta}\right)_{\eta=0}$.

The Nusselt number denoted by $N_{u}$ is proportional to $-\left(\frac{\partial T}{\partial y}\right)_{y=0}$, hence we have

$N_{u} \infty-\theta^{\prime}(0)$

The Sherwood number $S_{h}$ is proportional to $-\left(\frac{\partial C}{\partial y}\right)_{y=0}$, hence we have

$S_{h} \infty-\varphi^{\prime}(0)$.

The numerical values of the local skin-friction coefficients, the local Nusselt number and the local Sherwood number are sorted in Tables 1-3.

\section{Numerical Solution}

The set of non-linear ordinary differential equations (9)-(12) with boundary conditions (13) have been solved by using sixth order Runge-Kutta method along with the Nacthsheim-Swigert (1965) shooting iteration technique.

\section{Results and Discussion}

In this paper, the effect of different parameters entering into steady two-dimensional MHD free convection and mass transfer fluid flow through a porous medium past a continuously moving semiinfinite vertical porous plate $y=0$ in a rotating system has been investigated using NacthsheimSwigert shooting iteration technique.

For the purpose of discussing the effects of various parameters on the flow behavior, some numerical calculations have been carried out for non-dimensional primary velocity $f^{\prime}(\eta)$, secondary velocity $g(\eta)$, temperature $\theta(\eta)$ and concentration $\varphi(\eta)$. 


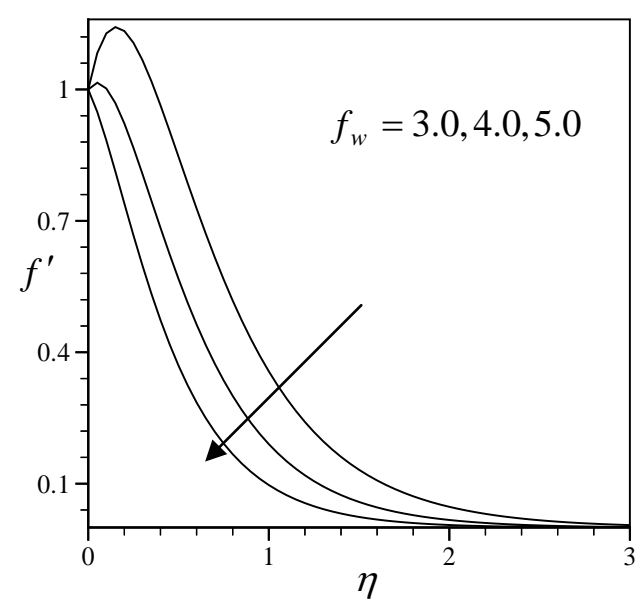

Fig. 2: Primary velocity profiles for different values of $f_{w}$ with $G_{r}=10.0, G_{m}=4.0$, $M=0.5, R=0.2, P_{r}=0.71, S_{r}=1.0$, $S_{c}=0.6, D_{f}=0.2, K=0.5$.

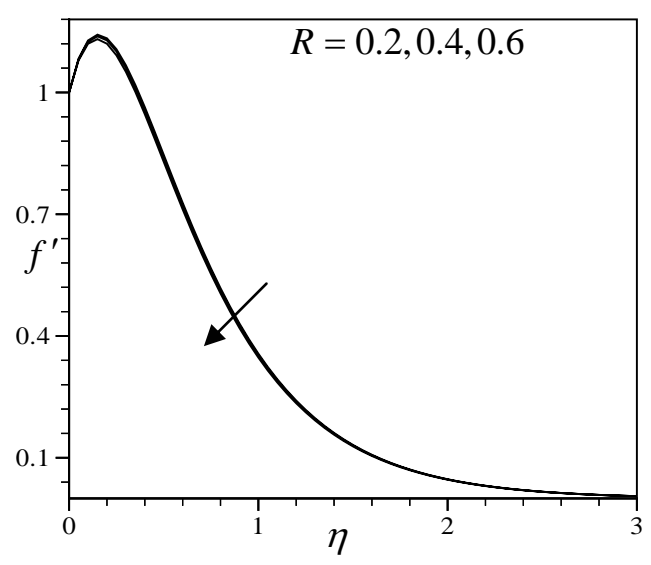

Fig. 4: Primary velocity profiles for different Fig. 5: Secondary velocity profiles for different values of $R$ with $f_{w}=3.0, G_{r}=10.0$,

$$
\begin{array}{lll}
G_{m}=4.0, & M=0.5, & P_{r}=0.71 \\
S_{r}=1.0, & S_{c}=0.6, & D_{f}=0.2 \\
K=0.5 .
\end{array}
$$

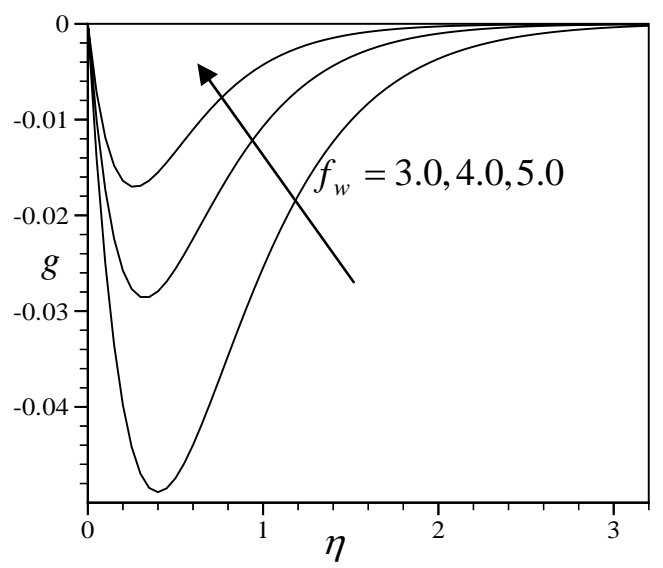

Fig. 3: Secondary velocity profiles for different values of $f_{w}$ with $G_{r}=10.0, G_{m}=4.0$, $M=0.5, R=0.2, P_{r}=0.71, S_{r}=1.0$, $S_{c}=0.6, D_{f}=0.2, K=0.5$.

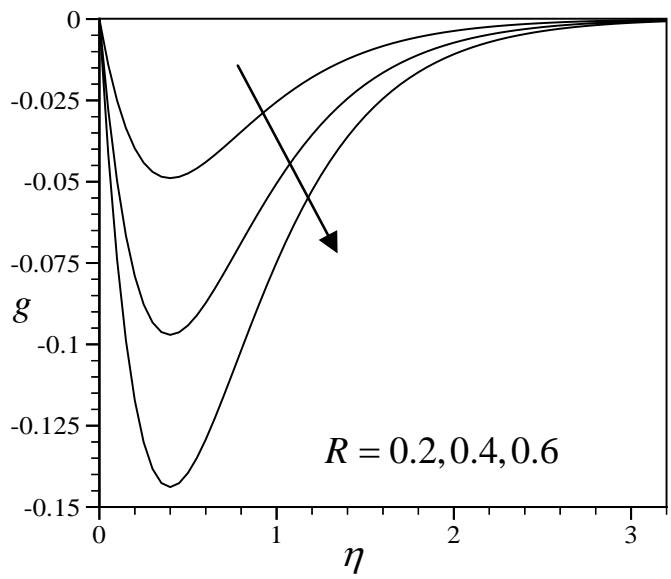
values of $R$ with $f_{w}=3.0, G_{r}=10.0$, $G_{m}=4.0, M=0.5, P_{r}=0.71, S_{r}=1.0$ , $S_{c}=0.6, D_{f}=0.2, K=0.5$.

The velocity profiles for $X$ and $z$ components of velocity, commonly known as non-dimensional primary $\left(f^{\prime}\right)$ and secondary $(g)$ velocities, are shown in Figs. 2 - 17 for different values of suction parameter $\left(v_{0}\right)$, the magnetic parameter $(M)$, the rotation parameter $(R)$, the Prandtl number $\left(P_{r}\right)$, the Soret number $\left(S_{r}\right)$, the Schmidt number $\left(S_{c}\right)$, the Dufour number $\left(D_{f}\right)$ and the permeability parameter $(K)$ and for fixed values of Grashof number $\left(G_{r}\right)$ and modified Grashof number $\left(G_{m}\right)$. 

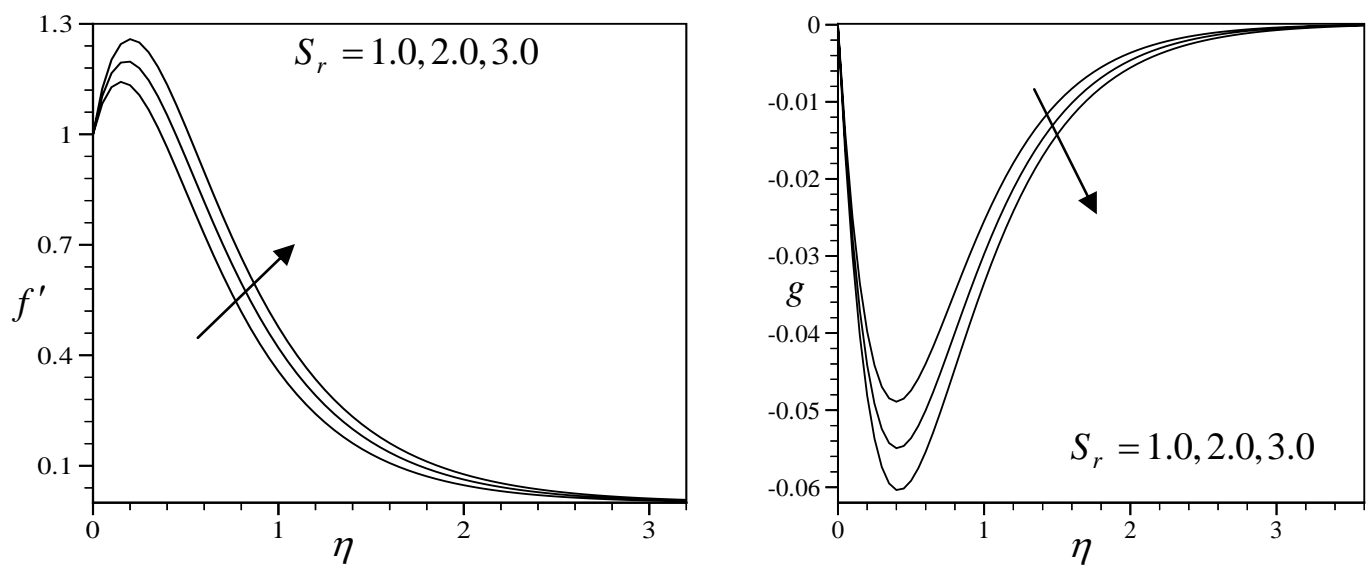

Fig. 6: Primary velocity profiles for different values Fig. 7: Secondary velocity profiles for different of $S_{r}$ with $f_{w}=3.0, G_{r}=10.0$, values of $S_{r}$ with $f_{w}=3.0$, $G_{m}=4.0 \quad, \quad M=0.5 \quad, \quad R=0.2$, $G_{r}=10.0, G_{m}=4.0, M=0.5, R=0.2$, $P_{r}=0.71, S_{c}=0.6, D_{f}=0.2$, $K=0.5$. $P_{r}=0.71, S_{c}=0.6, D_{f}=0.2$, $K=0.5$.
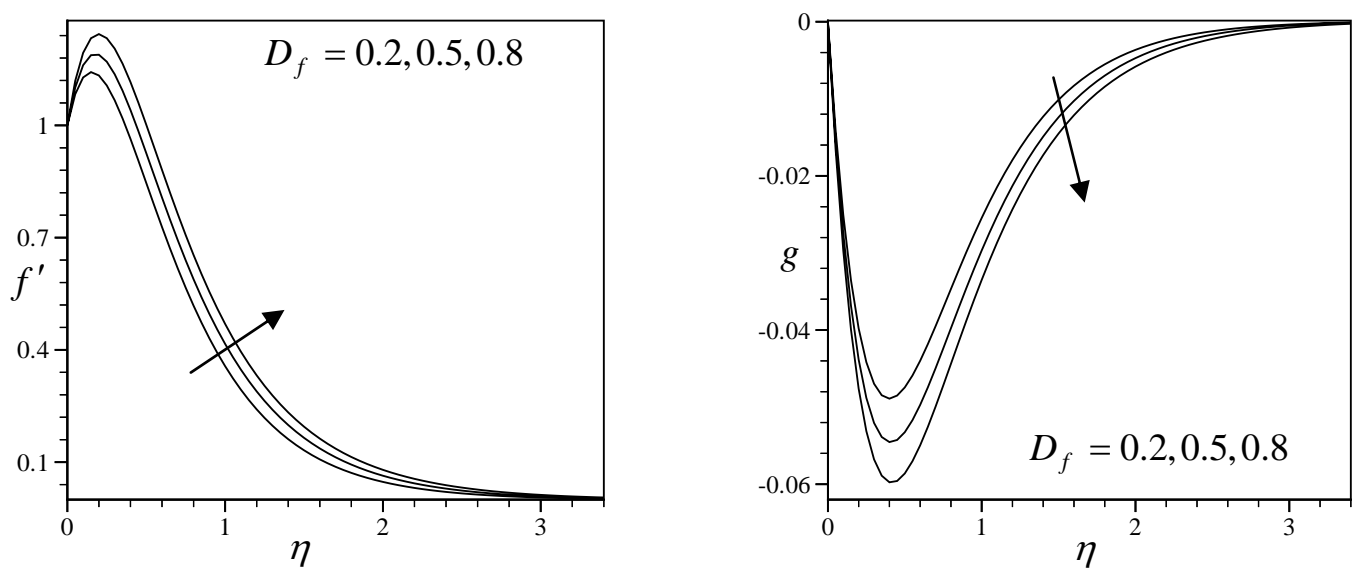

Fig. 8: Primary velocity profiles for Fig. 9: Secondary velocity profiles for different values of $D_{f}$ with $f_{w}=3.0$, different values of $D_{f}$ with

$$
\begin{array}{lll}
G_{r}=10.0 \quad, \quad G_{m}=4.0 \quad, & f_{w}=3.0, \quad G_{r}=10.0, G_{m}=4.0, \\
M=0.5, R=0.2, P_{r}=0.71, & M=0.5, R=0.2, P_{r}=0.71, S_{r}=1.0, \\
S_{r}=1.0, S_{c}=0.6, K=0.5 . & S_{c}=0.6, K=0.5 .
\end{array}
$$

For Prandtl number $\left(P_{r}\right)$, three values $0.71,1.0$ and 7.0 which represent air at 20 deg C, electrolytic solution such as salt water and water respectively. The values $0.22,0.60$ and 0.75 of the Schmidt number $\left(S_{c}\right)$ are also considered for they represent specific conditions of the flow. In particular, 0.22 corresponds to Hydrogen while 0.60 corresponds to water vapor that represents a diffusivity chemical species of most common interest in air and the value 0.75 represent Oxygen. In the calculations $f_{w}$, $M, R, S_{r}, D_{f}, K$ and $G_{m}$ are chosen arbitrarily. 


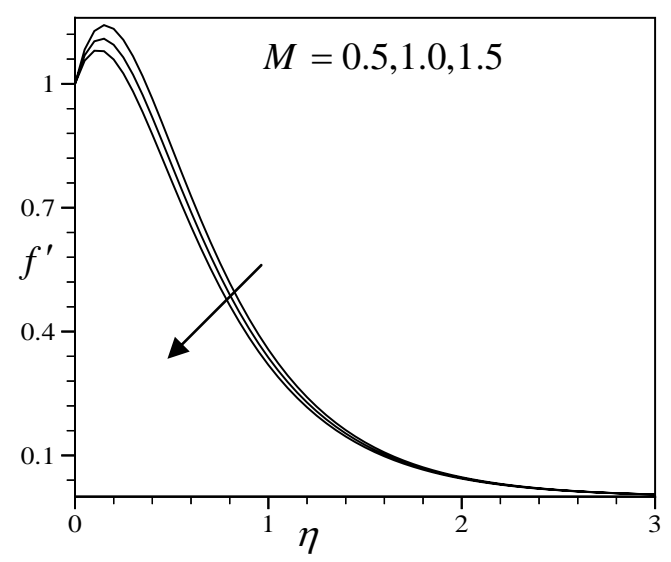

Fig. 10: Primary velocity profiles for different values of $M$ with $f_{w}=3.0, G_{r}=10.0, G_{m}=4.0$, $R=0.2 \quad, \quad P_{r}=0.71 \quad, \quad S_{r}=1.0$, $S_{c}=0.6, D_{f}=0.2, K=0.5$.

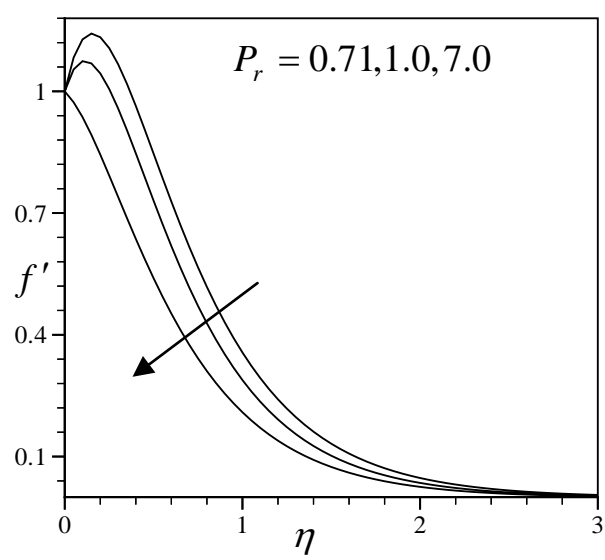

Fig. 12: Primary velocity profiles for different values of $P_{r}$ with $f_{w}=3.0$, $G_{r}=10.0, G_{m}=4.0, M=0.5$, $R=0.2 \quad, \quad S_{r}=1.0 \quad, \quad S_{c}=0.6 \quad$, $D_{f}=0.2, K=0.5$.

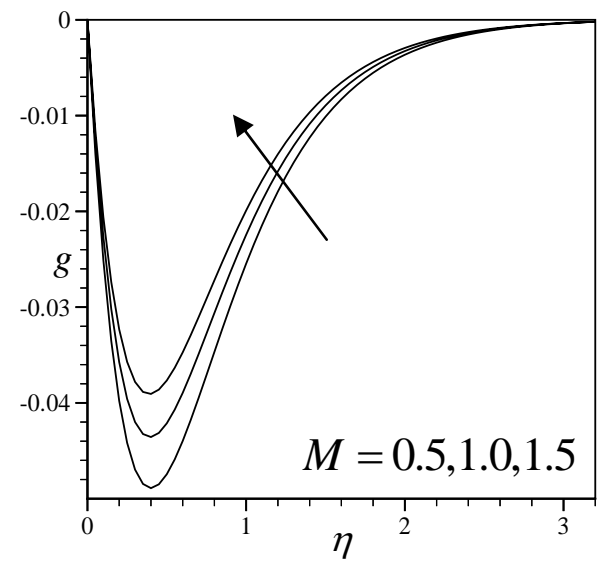

Fig. 11: Secondary velocity profiles for different values of $M \quad$ with $f_{w}=3.0 \quad, \quad G_{r}=10.0$ $G_{m}=4.0 \quad, \quad R=0.2$ $P_{r}=0.71 \quad, \quad S_{r}=1.0$ $S_{c}=0.6, D_{f}=0.2, K=0.5$.

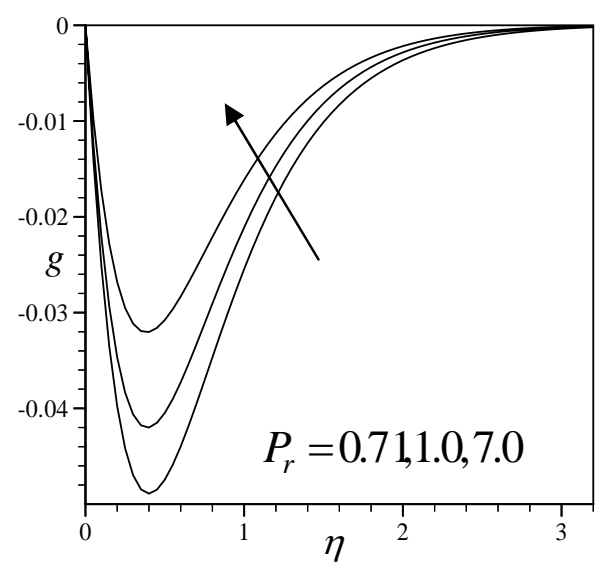

Fig. 13: Secondary velocity profiles for different values of $P_{r}$ with $f_{w}=3.0$, $G_{r}=10.0, G_{m}=4.0, M=0.5$, $R=0.2 \quad, \quad S_{r}=1.0 \quad, \quad S_{c}=0.6 \quad$, $D_{f}=0.2, K=0.5$.

With the above mentioned flow parameters, it is observed from Figs. 2 and 3 that an increase in the suction parameter $f_{w}$ leads to decrease in the primary velocity and to increase the secondary velocity.

The variations of the primary and secondary velocities for different values of rotation parameter $R$ are shown in Figs. 4 \& 5. From these figures it is observed that the rotation parameter $R$ has a minor decreasing effect on the primary velocity while it has quite a larger decreasing effect on the secondary velocity. In Figs. $6 \& 7$ and $8 \&$ 9, the variations of the primary and secondary velocities for different 
values of Soret number $S_{r}$ and Dufour number $D_{f}$ are shown respectively. From these figures it is observed that the primary velocity increases with the increase of Soret number $S_{r}$ and Dufour number $D_{f}$. The effects of Soret number $S_{r}$ and Dufour number $D_{f}$ on the secondary velocity are opposite to that of the primary velocity. In Figs. $10 \& 11,12 \& 13,14 \& 15$ and $16 \& 17$, the variations of the primary and secondary velocities for different values of magnetic parameter $M$, Prandtl number $P_{r}$, Schmidt number $S_{c}$ and permeability parameter $K$ are shown respectively. It is observed from these figures that the magnetic parameter $M$ has a decreasing effect on the primary velocity and increasing effect on the secondary velocity. It is also seen from these figures that the primary velocity decreases while the secondary velocity increases with the increase of Prandtl number $P_{r}$. The same effects are observed in the case of Schmidt number $S_{c}$ and permeability parameter $K$. The effects of various parameters on non-dimensional temperature are shown in Figs. 18-20. It is observed from Fig. 18 that the temperature decreases with the increase of suction parameter $f_{w}$ increase. In Fig. 19, the temperature profiles for different values of Dufour number $D_{f}$ are shown. It is observed from this figure that the Dufour number $D_{f}$ has an increasing effect. In Fig. 20, the temperature profiles for different values of Prandtl number $P_{r}$ are shown. This figure revels that the Prandtl number $P_{r}$ has a large decreasing effect on temperature.The effects of various parameters on the concentration field are shown in Figs. 21 - 24. It is observed from Fig. 21 that the concentration decreases as the suction parameter $f_{w}$ increase. The concentration profiles for different values of Soret number $S_{r}$ are shown in Fig. 22. The figure shows that the concentrations increases as the Soret number $S_{r}$ increase.

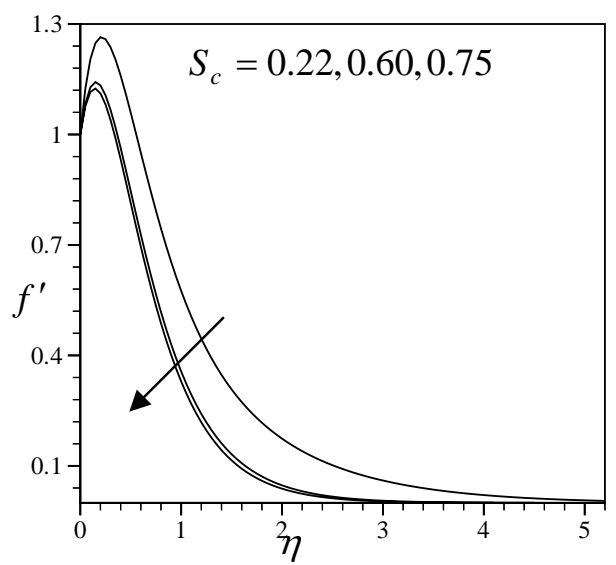

Fig. 14: Primary velocity profiles for different values of $S_{c}$ with $f_{w}=3.0$, $G_{r}=10.0, G_{m}=4.0, M=0.5$, $R=0.2, \quad P_{r}=0.71, \quad S_{r}=1.0$, $D_{f}=0.2, K=0.5$.

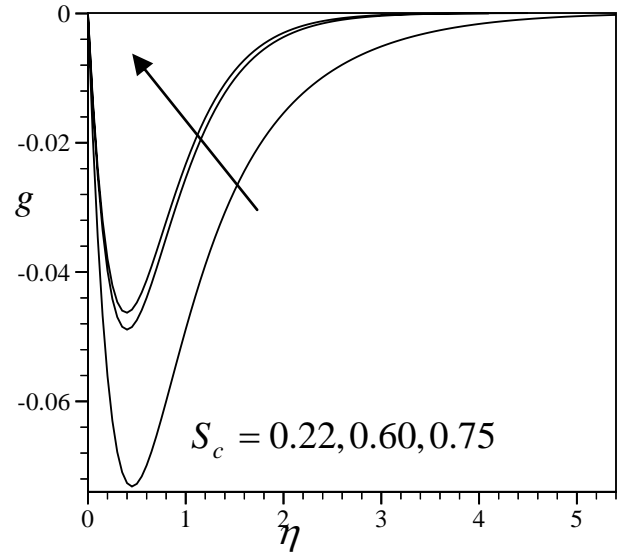

Fig. 15: Secondary velocity profiles for different values of $S_{c}$ with $f_{w}=3.0$, $G_{r}=10.0, G_{m}=4.0, M=0.5$, $R=0.2 \quad, \quad P_{r}=0.71 \quad, \quad S_{r}=1.0$, $D_{f}=0.2, K=0.5$.

In Figs. 23 and 24, the concentration profiles for different values of Prandtl number $P_{r}$ and Schmidt number $S_{c}$ are shown respectively. It is observed from these figures that the concentration increases as the Prandtl number $P_{r}$ increase while the concentration decreases as the Schmidt number $S_{c}$ increase. Finally, the effects of various parameters on the components of the skin friction coefficient $\tau_{x}$ and $\tau_{z}$, the Nusselt number $N_{u}$ and the Sherwood number $S_{h}$ are shown in Tables 1-3. 

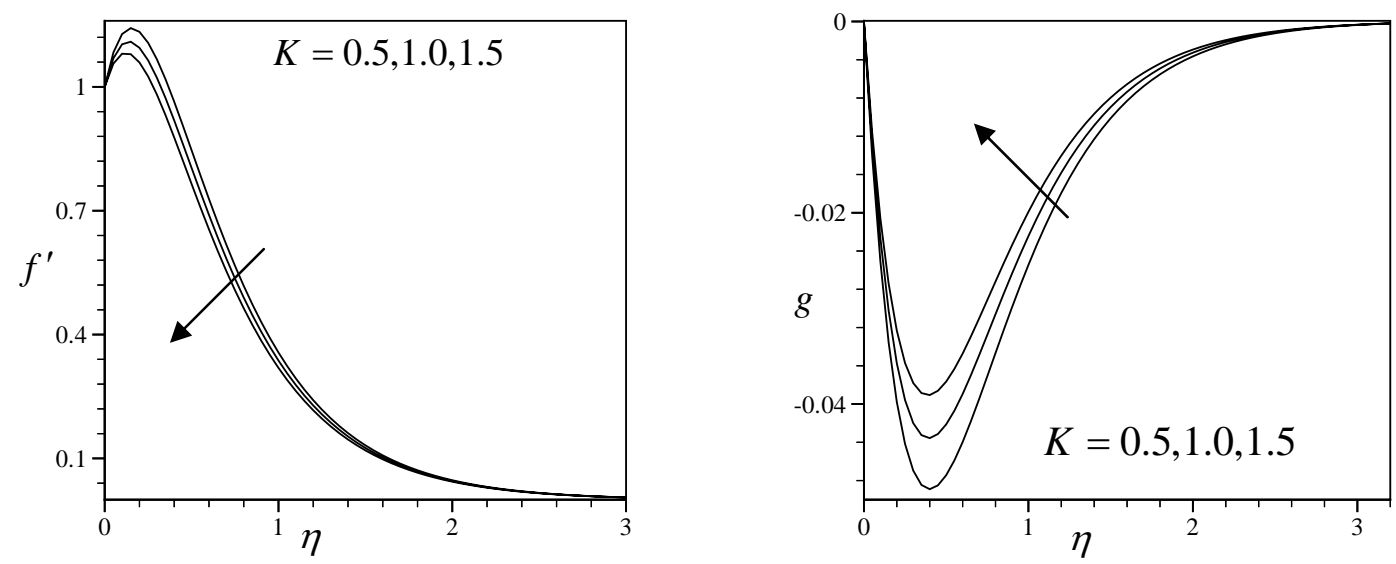

Fig. 16: Primary velocity profiles for different Fig. 17: Secondary velocity profiles for different values of $K$ with $f_{w}=3.0, G_{r}=10.0$, values of $K$ with $f_{w}=3.0, G_{r}=10.0$, $G_{m}=4.0, \quad M=0.5, \quad R=0.2$, $G_{m}=4.0, \quad M=0.5, \quad R=0.2$, $P_{r}=0.71, S_{r}=1.0, S_{c}=0.6$, $P_{r}=0.71, S_{r}=1.0, \quad S_{c}=0.6$, $D_{f}=0.2$. $D_{f}=0.2$

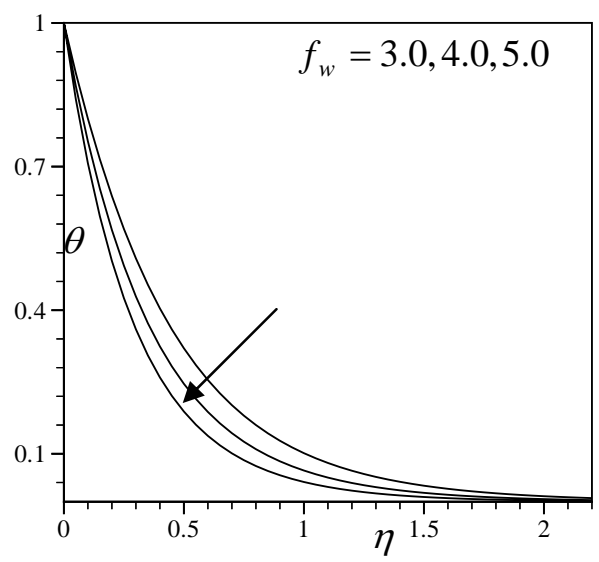

Fig. 18: Temperature profiles for different values of $f_{w}$ with $G_{r}=10.0, G_{m}=4.0$, $M=0.5, R=0.2, P_{r}=0.71$, $S_{r}=1.0, S_{c}=0.6, D_{f}=0.2$, $K=0.5$.

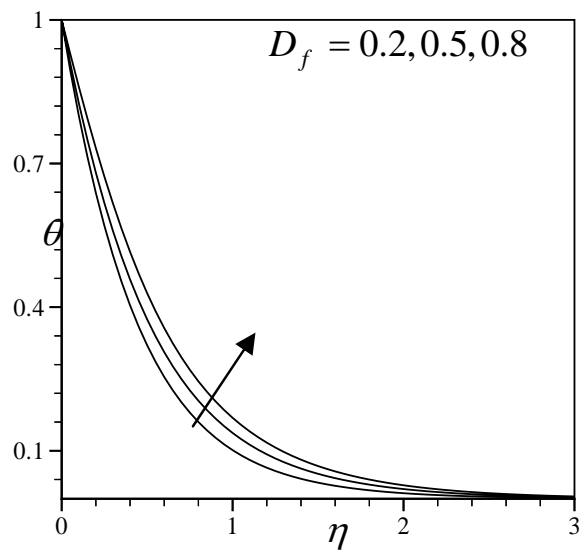

Fig. 19: Temperature profiles for different values of $D_{f}$ with $f_{w}=3.0, G_{r}=10.0$, $G_{m}=4.0, M=0.5 \quad, \quad R=0.2$, $P_{r}=0.71, S_{r}=1.0, S_{c}=0.6$, $K=0.5$.

From Table 1, we observe that the skin-friction component $\tau_{x}$ decreases with the increase of suction parameter $f_{w}$, but the skin-friction component $\tau_{z}$, the Nusselt number $N_{u}$ and the Sherwood number $S_{h}$ increase with the increase of suction parameter $f_{w}$. It is also observed from this table that the skin-friction component $\tau_{x}$, the Nusselt number $N_{u}$ and Sherwood number $S_{h}$ decrease with the increase of magnetic parameter $M$, while the skin-friction component $\tau_{z}$ increases with the increase of magnetic parameter $M$. 
Again, from Table 2, we observe that the skin-friction components $\tau_{x}$ and $\tau_{z}$, the Nusselt number $N_{u}$ and the Sherwood number $S_{h}$ decrease with the increase of rotation parameter $R$. It is also observed from this table that the skin-friction component $\tau_{x}$ and the Nusselt number $N_{u}$ increase while the skin-friction component $\tau_{z}$ and the Sherwood number $S_{h}$ decrease with the increase of Soret number $S_{r}$.

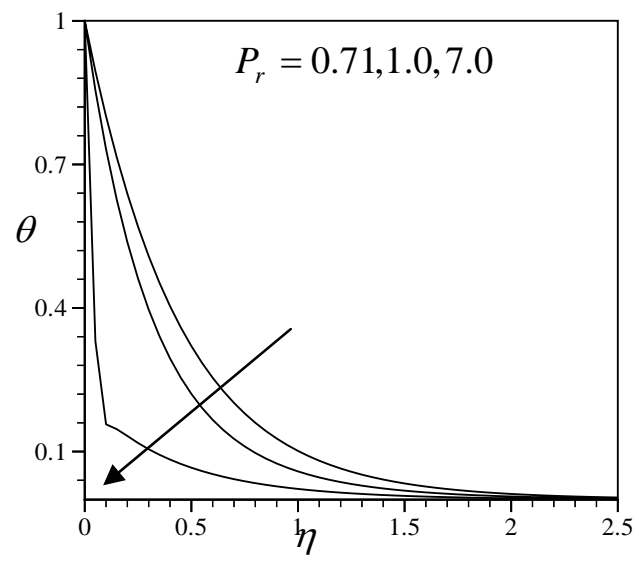

Fig. 20: Temperature profiles for different values of $P_{r}$ with $f_{w}=3.0, G_{r}=10.0$, $G_{m}=4.0, \quad M=0.5, \quad R=0.2$, $S_{r}=1.0, S_{c}=0.6, D_{f}=0.2$, $K=0.5$.

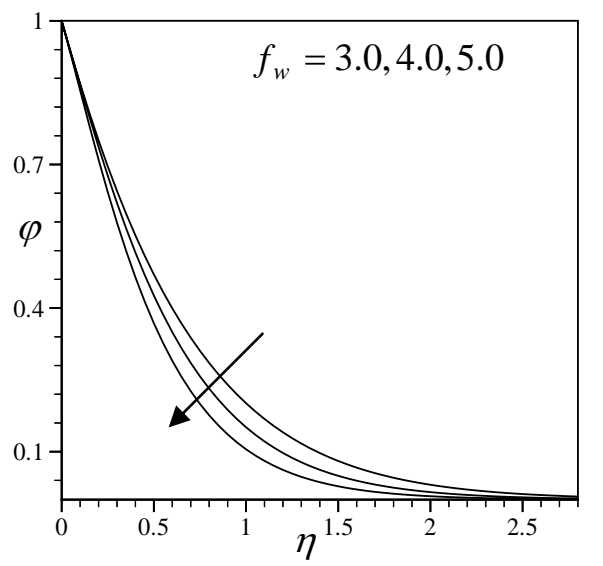

Fig. 21: Concentration profiles for different values of $f_{w}$ with $G_{r}=10.0, G_{m}=4.0$, $M=0.5 \quad, \quad R=0.2 \quad, \quad P_{r}=0.71$, $S_{r}=1.0 \quad, \quad S_{c}=0.6 \quad, \quad D_{f}=0.2$, $K=0.5$.

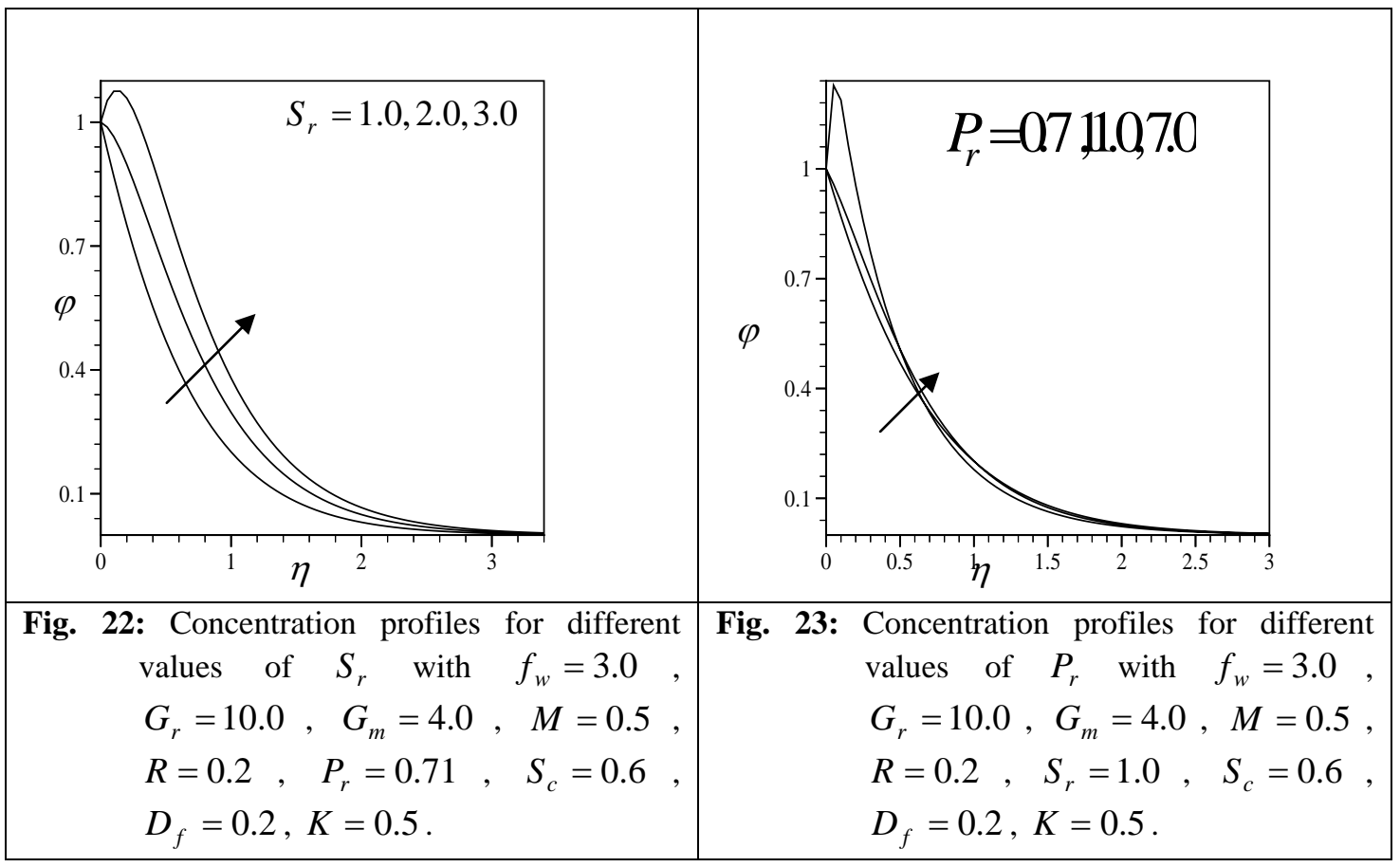




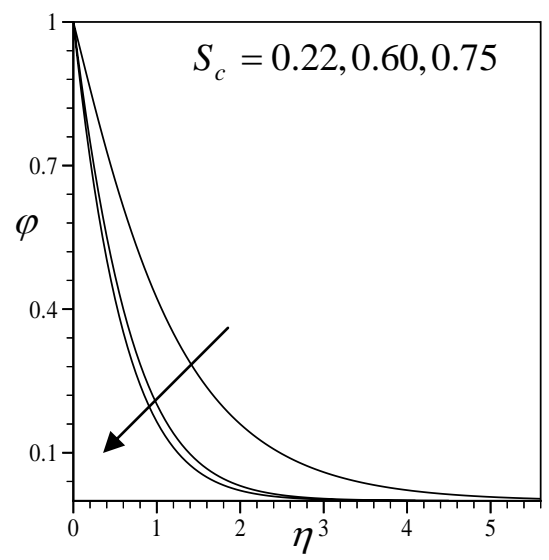

From Table 3, we observe that the skin-friction component $\tau_{x}$ and the Sherwood number $S_{h}$ increase while the skin-friction component $\tau_{z}$ and the Nusselt number $N_{u}$ decrease owing to the increase of Dufour number $D_{f}$. It is also seen from this table that the skin-friction component $\tau_{x}$, the Nusselt number $N_{u}$ and the Sherwood number $S_{h}$ decrease while the skin-friction component $\tau_{z}$ increases with the increase of permeability parameter $K$.

Fig. 24: Concentration profiles for different values of $S_{c}$ with $f_{w}=3.0$, $G_{r}=10.0, G_{m}=4.0, M=0.5$, $R=0.2, \quad P_{r}=0.71, \quad S_{r}=1.0$ ， $D_{f}=0.2, K=0.5$.

Table 1: Numerical values of $\tau_{x}, \tau_{z}, N_{u}$ and $S_{h}$ for $P_{r}=0.71, G_{r}=10.0, G_{m}=4.0$, $R=0.2, S_{r}=1.0, S_{c}=0.6, D_{f}=0.2$ and $K=0.5$.

\begin{tabular}{|c|c|c|c|c|c|}
\hline$f_{w}$ & $M$ & $\tau_{x}$ & $\tau_{z}$ & $N_{u}$ & $S_{h}$ \\
\hline 3.0 & 0.5 & 2.1183684 & -0.3123093 & 2.2126436 & 1.3606514 \\
\hline 4.0 & 0.5 & 0.6630225 & -0.2322516 & 2.8415285 & 1.3736072 \\
\hline 5.0 & 0.5 & -0.8398383 & -0.1720828 & 3.4941526 & 1.3843751 \\
\hline 3.0 & 1.0 & 1.8034374 & -0.2837792 & 2.2074190 & 1.3379786 \\
\hline 3.0 & 1.5 & 1.5115707 & -0.2596410 & 2.2026285 & 1.3168868 \\
\hline
\end{tabular}

Table 2: Numerical values of $\tau_{x}, \tau_{z}, N_{u}$ and $S_{h}$ for $f_{w}=0.5, G_{r}=10.0, G_{m}=4.0$, $M=0.5, P_{r}=0.71, S_{c}=0.6, D_{f}=0.2$ and $K=0.5$.

\begin{tabular}{|c|c|c|c|c|c|}
\hline$R$ & $S_{r}$ & $\tau_{x}$ & $\tau_{z}$ & $N_{u}$ & $S_{h}$ \\
\hline 0.2 & 1.0 & 2.1183684 & -0.3123093 & 2.2126436 & 1.3606514 \\
\hline 0.4 & 1.0 & 2.0825316 & -0.6208549 & 2.2119861 & 1.3572153 \\
\hline 0.6 & 1.0 & 2.0241833 & -0.9220832 & 2.2107869 & 1.3517746 \\
\hline 0.2 & 2.0 & 2.5626587 & -0.3425054 & 2.4041973 & 0.1109341 \\
\hline 0.2 & 3.0 & 2.9919283 & -0.3699451 & 2.6305160 & -1.3989729 \\
\hline
\end{tabular}

Table 3: Numerical values of $\tau_{x}, \tau_{z}, N_{u}$ and $S_{h}$ for $f_{w}=0.5, G_{r}=10.0, G_{m}=4.0$, $P_{r}=0.71, R=0.2, M=0.5, S_{r}=1.0$ and $S_{c}=0.6$.

\begin{tabular}{|c|c|c|c|c|c|}
\hline$D_{f}$ & $K$ & $\tau_{x}$ & $\tau_{z}$ & $N_{u}$ & $S_{h}$ \\
\hline 0.2 & 0.5 & 2.1183684 & -0.3123093 & 2.2126436 & 1.3606514 \\
\hline 0.5 & 0.5 & 2.5017350 & -0.3402315 & 1.8900020 & 1.5829858 \\
\hline 0.8 & 0.5 & 2.8887150 & -0.3663611 & 1.4347118 & 1.8786190 \\
\hline 0.2 & 1.0 & 1.8034374 & -0.2837792 & 2.2074190 & 1.3379786 \\
\hline 0.2 & 1.5 & 1.5115707 & -0.2596410 & 2.2026285 & 1.3168868 \\
\hline
\end{tabular}




\section{References}

Alam, M. S. and Rahman, M. M. (2006): Dufour and Soret Effects on Mixed Convection Flow past a Vertical Porous Flat Plate with Variable Suction, Nonlinear analysis: Modeling and Control, 11, 1.

Anghel, M., Takhur, H. S. and Pop, I. (2000): Dufour and Soret Effects on Free Convection Boundary-layer over a Vertical Surface Embedded in a Porous Medium, Studia Universitatis Babes-Bolyai. Mathematica, XLV(4), 11.

Eckert, E. R. G. and Drake, R. M. ( 1972): Analysis of Heat and Mass Transfer, McGraw-Hill Book Co., New York.

Georgantopoulos, G. A. (1979): Effects of Free Convection on the Hydromagnetic Accelerated Flow Past a Vertical Porous Limiting Surface. Astrophysics and Space Science, 65, 433.

Gebhart, B. and Pera, L. (1971): The Nature of Vertical Convection Flows Resulting from Combined Buoyancy Effects of the Thermal and Mass Diffusion Nature, International Journal of Heat and Mass Transfer, 14, 2025.

Kafousias, N. G., Nanousis, N. and Georgantopoulos, G. A. (1979): Free Convection Effects on the Stokes Problem for an Infinite Vertical Limiting Surface with Constant Suction, Astrophysics and Space Science, 64, 391.

Kafoussias, N. G. and Williams, E. M. (1995): Thermal-diffusion and Diffusion-thermo Effects on Mixed Free-forced Convective and Mass Transfer Boundary Layer Flow with Temperature Dependent Viscosity, Internatinal Journal of Engineering Science, 33, 1369.

Nachtssheim, P. R. and Swigert, P. (1965): Satisfaction of the Asymptotic Boundary Conditions in Numerical Solution of the System of Nonlinear Equations of Boundary Layer Type, NASA TND-3004.

Nanousis, N., Georgantopoulos, G. A. and Papaioannou, A. (1980): Hydromagnetic Free Convection Flow in the Stokes Problem for a Porous Vertical Limiting Surface with Constant Suction, Astrophysics and Space Science, 70, 377.

Pai, S. I. (1962): Magnetogasdynamics and Plasma dynamics, Springier Verlag, NewYork.Raptis, A. and Singh, A. K. (1983): MHD Free Convection Flow Past an Accelerated Vertical Plate, International Communications in Heat and Mass Transfer, 10, 313.

Raptis, A. and Singh, A. K. (1985): Rotation Effects on MHD Free Convection Flow Past an Accelerated Vertical Plate, Mechanical Resulation Communication, 12, 31.

Singh, A. K. (1982): MHD Free Convection Flow in the Stokes Problem for a Porous Vertical Plate, Astrophysics and Space Science, 87, 455.

Singh, A. K. and Singh, J. N. (1989): Transient MHD Free Convection in a Rotating System, Astrophysics and Space Science, 162, 85.

Singh, A. K. (1983): MHD Free Convection Flow in the Stokes Problem for a Vertical Porous Plate in a Rotating System, Astrophysics and Space Science, 95, 283.

Singh, A. K. (1984a): Hydromagnetic Free Convection Flow Past an Impulsively Started Vertical Plate in a Rotating System, International Communications in Heat and Mass Transfer, 11, 349.

Singh, A. K. (1984b): Stokes Problem for a Porous Vertical Plate with Heat Sinks by Finite Difference Method, Astrophysics and Space Science, 103, 55. 